This document is the unedited Author's version of a Submitted Work that was subsequently accepted for publication in 'The Journal of Physical Chemistry C', copyright @ A American Chemical Society after peer review. To access the final edited and published work: https://pubs.acs.org/doi/abs/10.1021/sc500479b

\title{
Development of a Recyclable Remediation System for Gaseous BTEX: Combination of Iron Oxides Nanoparticles Adsorbents and Electrochemistry
}

\author{
Zhenzhong Hu, Maximilien Beuret, Hassan Khan, and Parisa A. Ariya
}

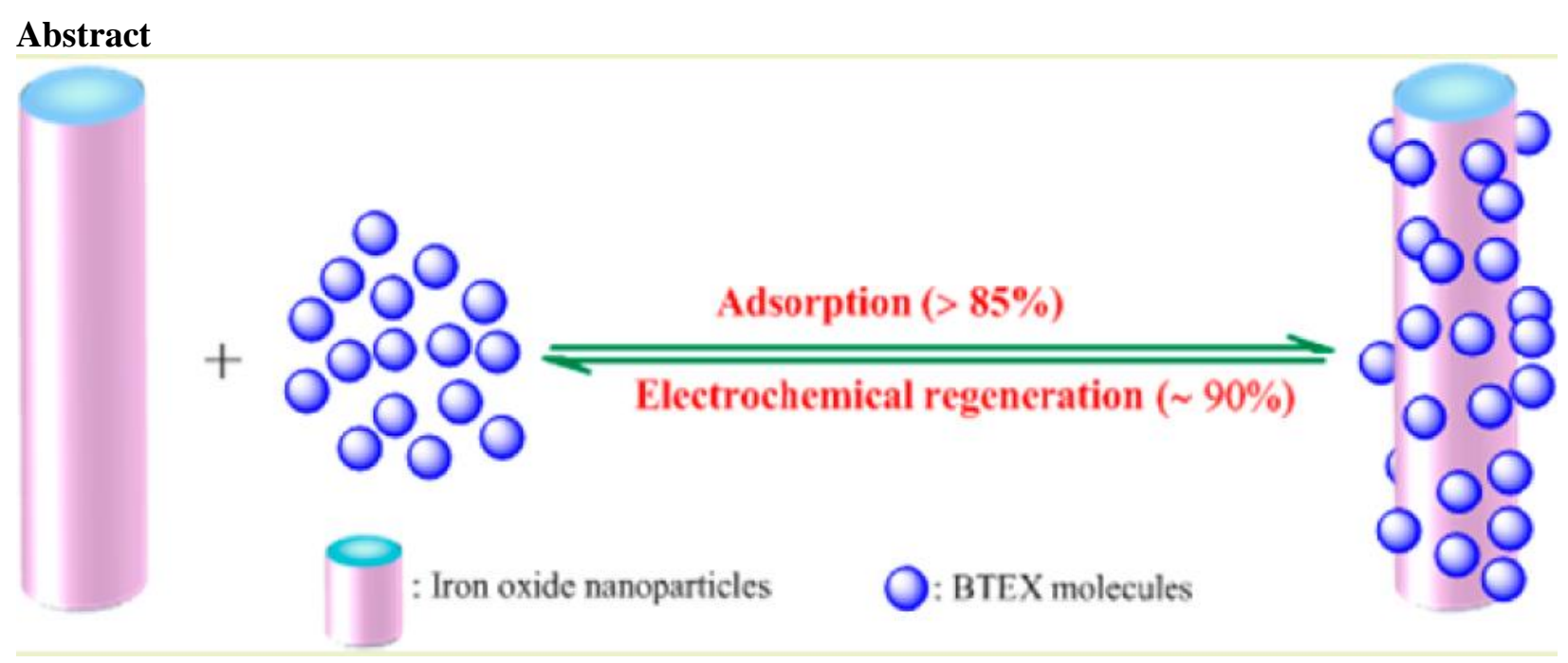

We designed a two-step green technique to remove and recycle selected gaseous air pollutants. The first step includes the assessment of adsorption efficiencies of BTE (benzene, toluene, ethylbenzene, and xylenes) on magnetite, hematite, and their composite surfaces. Improvement of the synthesis method led to BTEX adsorption (>85\%; 200 ppmv) on $1.0 \mathrm{~g}$ of nanoparticles within a time scale of minutes. The second element included the design of an electrochemical reactor for the regeneration of used nanoparticles. $\mathrm{NaOH}$ showed superior performance as an electrolyte in comparison to $\mathrm{NaCl}$ and $\mathrm{Na}_{2} \mathrm{CO}_{3}$. The stripping efficiency for cathodic regeneration was higher than the anodic one. Under optimized conditions, the stripping efficiency was up to $85 \%$. Iron oxides nanoparticles were regenerated $(\sim 90 \%)$. Using high-resolution transmission electron microscopy, X-ray diffraction, NanoScan, and Brunauer- Emmett- Teller, selected physical and chemical properties of nanosurfaces were analyzed, revealing that the physical properties of nanoparticles remained unchanged during the regeneration process.

\section{Introduction}

During the last decades, nanoparticles have been used for a wide range of pollution remediation studies. Due to their high surface area to mass ratio of nanoparticles, they can exhibit excellent sorbent adsorption capabilities for selected pollutants. Naturally occurring nanoparticles, such as magnetite and hematite, are ubiquitous components of airborne dust particles, which are also usually low in toxicity, abundant, and bioactive. They have played an important role in limiting and controlling certain trace pollutants. ${ }^{1} \mathrm{We}$, along with other research groups, have demonstrated that titanium dioxide and iron oxides nanoparticles are effective in removing trace metal pollutants and volatile air pollutants, respectively. ${ }^{2,3}$ Due to their magnetic properties, magnetite nanoparticles can act as promising sorbents because they can rapidly be separated and by applying an appropriate magnetic field can be transported. Magnetite nanoparticles have been broadly used for adsorption of contaminants in groundwater or contaminated land. ${ }^{4,5}$ However, little research has been conducted on the gaseous pollutants' remediation using $\mathrm{Fe}_{3} \mathrm{O}_{4}$ nanoparticles. ${ }^{3}$ Magnetite can also be simply and more environmentally friendly synthesized to avoid generating hazardous wastes; ${ }^{6,7}$ therefore, it is appropriate to develop an environmental and sustainable method for gaseous pollutant remediation using recyclable magnetite nanoparticles.

BTEX (benzene, toluene, ethylbenzene, and xylenes) are widely used as model molecules to represent volatile organic compounds (VOCs). BTEX are commonly found in crude petroleum, coal tar, and a wide range of petroleum products. ${ }^{8}$ These monoaromatic hydrocarbons are significant environ- mental concerns to the ecosystem, ${ }^{9}$ and 
This document is the unedited Author's version of a Submitted Work that was subsequently accepted for publication in 'The Journal of Physical Chemistry C', copyright () American Chemical Society after peer review. To access the final edited and published work: https://pubs.acs.org/doi/abs/10.1021/sc500479b

exposure to these VOCs can cause serious harm to human health, including neurological and respiratory damage, and can damage the nervous system. ${ }^{10}$ Most VOCs are directly released into the atmosphere, play important roles in atmospheric chemistry, and have been linked to organic aerosols and ozone formation processes,,${ }^{11,12}$ as well as climate change through interactions with radiation. ${ }^{13}$ Typically released from gasoline, diesel fuel, and tobacco smoke, a large quantity of BTEX compounds is also released from various industrial plants and processes. BTEX continue to be used extensively and persistently in industrial applications despite proven toxic properties. These reasons are driving researchers to develop efficient methods to reduce emissions and enhance their recovery. Materials such as activated carbon, carbon nanotubes, zeolites, and mesoporous silica have been suggested as adsorbents in treating BTEX compounds. ${ }^{14}$ On large scales, there are challenges such as low selectivity, complexity, and high cost of synthesis ${ }^{15,16}$ associated with applying these adsorbents for BTEX remediation, and thesechallenges are prompting us toexplore new sorbents such as magnetite, hematite, and their composites to remove BTEX compounds.

To reduce the economic and environmental impacts, exhausted nanoparticles upon pollutant absorption have to be efficiently regenerated. The most widely used methods include thermal and chemical regeneration, but there are key disadvantages with these conventional methods. The thermal regeneration method requires high energy cost to obtain high efficiency, and nanoparticles lose considerable weight, especially after several recycling circles. ${ }^{17}$ In chemical regeneration processes, the drawbacks are the introduction of extra waste and possibly pore damage to the nanoparticles as well as the typical regeneration efficiency being lower than $70 \% .{ }^{18}$ Therefore, a more economical, sustainable, and more efficient regeneration method is warranted, and electrochemical regeneration has proven to be an alternative promising method for the regeneration of nanoparticles sorbents. In recent years, successful research has been undertaken for the electrochemical regeneration of exhausted activated carbon. ${ }^{19-22}$ Except for the

high regeneration efficiency obtained, the porosity of the nanoparticles was highly recovered.

We herein developed a two-part pollution remediation system for gaseous BTEX removal on a suite of iron nanoparticles followed by electrochemical regeneration of nanoparticles (macro-size upon coagulation) saturated with BTEX compounds. To our knowledge, this is the first such study leading to the development of an efficient, consistent, and cost-effective electrochemical regeneration system for iron oxides nanoparticles. Different types of magnetite, hematite, and their mixture of adsorbents, as well as a range of electrolytes, electrolysis current, and time, were assessed, and the complete system was optimized

\section{Experimental methods}

Materials. Sodium chloride, anhydrous sodium carbonate, and sodium hydroxide pellets were used as purchased. Commercial magnetite and hematite nanoparticles were purchased from Sigma- Aldrich without further purification. Platinum gauze (52 mesh) and copper wire $(20 \mathrm{oz})$ were used. For the electrolyte solutions, ultrapure water (Millipore Milli-Q;18.2 M Ohm cm) was employed.

Synthesis and Characterization of $\mathrm{Fe}_{3} \mathrm{O}_{4}\left(\mathrm{Fe}_{2} \mathrm{O}_{3} \cdot \mathrm{FeO}\right)$ Nano- particles. The Massart ${ }^{23}$ method was followed wherein the $\mathrm{Fe}_{3} \mathrm{O}_{4} \mathrm{NPs}$ were precipitated by mixing a 2:1 solution of $\mathrm{FeCl}_{3} \cdot 6 \mathrm{H}_{2} \mathrm{O}$ and

$\mathrm{FeCl}_{2} \cdot 4 \mathrm{H}_{2} \mathrm{O}$ with ammonium hydroxide in $80-90{ }^{\circ} \mathrm{C}$ deoxygenated water. The formation of $\mathrm{Fe}_{3} \mathrm{O}_{4}$ is briefly described in eq 1 . In order to

get small-sized nanoparticles and narrow-sized distributions, we simply modified the synthesis method. First, we add $1 \%$ hydrochloride acid to hydrolyze the iron solutions to get a better mix. Then, we increased the reaction time at $90{ }^{\circ} \mathrm{C}$ with vigorous stirring. In order to efficiently remove the byproducts of ammonium chloride, we used warm deoxygenated water to wash the suspension. Meanwhile, we put the suspension into a $120^{\circ} \mathrm{C}$ oven for a short time to quickly remove the water and to efficiently avoid aggregation. The resulting beads were collected with a magnetic retriever and dried in a $50^{\circ} \mathrm{C}$ air ventilated oven.

$$
2 \mathrm{Fe}^{3+}+\mathrm{Fe}^{2+}+8 \mathrm{OH}^{-} \underset{\text { stir }}{\stackrel{80-90^{\circ} \mathrm{C}}{\rightarrow}} \mathrm{Fe}_{3} \mathrm{O}_{4}+4 \mathrm{H}_{2} \mathrm{O}
$$

Several complementary analytical techniques were used to characterize the $\mathrm{Fe}_{3} \mathrm{O}_{4}$ nanoparticles. High-resolution transmission electron microscopy (HR-TEM) was recorded on a Philips CM200 TEM operating at $200 \mathrm{kV}$. X-ray diffraction (XRD) patterns were analyzed on a Siemens D5000 diffractometer with a $\mathrm{Cu} K \alpha$ radiation source $(\lambda=$ $1.5418 \AA$ ). The Brunauer-Emmett-Teller (BET) specific surface area (SSA) was obtained by a nitrogen adsorption method on a TriStar $3000 \mathrm{~V} 6.07$ surface area analyzer at $77 \mathrm{~K}$. The particle size distribution of $\mathrm{Fe}_{3} \mathrm{O}_{4}$ nanoparticles in water suspension was measured by nanoparticle tracking analysis (NTA; Malvern NanoSight NS500). Magnetic properties of $\mathrm{Fe}_{3} \mathrm{O}_{4}$ nanoparticles were characterized at room temperature using a vibrating sample magnetometer (VSM). 
This document is the unedited Author's version of a Submitted Work that was subsequently accepted for publication in 'The Journal of Physical Chemistry C', copyright () American Chemical Society after peer review. To access the final edited and published work: https://pubs.acs.org/doi/abs/10.1021/sc500479b

Iron Oxides Nanoparticles Loaded with BTEX. For toluene adsorption experiments, the magnetite nanoparticles were loaded using the Schlenk technique. Toluene and other BTEX stock solutions were freshly made using vacuum transfer. Three-necked round-bottomed flasks were coated with Glassclad-18 (P200), and magnetite or hematite nanoparticles or the mixtures were subsequently inserted into the flask and evacuated/refilled three times with dry air to atmospheric pressure. The gas stock solution was then injected into the round- bottomed flasks to reach a $200 \mathrm{ppmv}$ mixing ratio. The absorption experiments were conducted in a room where the temperature was maintained constant at $25 \pm 3{ }^{\circ} \mathrm{C}$.

Regarding $\mathrm{Fe}_{3} \mathrm{O}_{4}$ NPs, upon BTEX adsorption for the electro- chemical regeneration, the adsorption follows the same process mentioned above. We use $1.0 \mathrm{~g}$ of magnetite for each BTEX loading. After the adsorption reaches equilibrium, the $2 \mathrm{~L}$ flask is immersed in liquid nitrogen for $30 \mathrm{~min}$. Adsorbed BTEX compounds can then be kept frozen into $\mathrm{Fe}_{3} \mathrm{O}_{4}$ NPs to avoid releasing into air during the shift process. $\mathrm{Fe}_{3} \mathrm{O}_{4}$ NPs upon BTEX adsorption were quickly transferred into the electrolysis cell by a magnet attached to the electrode.

Experimental Setup for Electrolysis. The electrolysis experi- ments were performed in a $3 \mathrm{~L}$ electrochemical glass cell. Platinum gauze ( $52 \mathrm{mesh})$ and copper wire $(20 \mathrm{oz})$ were used for the electrodes. Ultrapure water was used for the preparation of the electrolyte solutions. Figure S1 of the Supporting Information shows the electrochemical regeneration apparatus, which consisted of a glass regeneration reactor and a power supply. The DC power was supplied by HQ PowerTM (PS1503SBU). The regeneration reactor consisted of a $22 \mathrm{~cm}$ high and $13.2 \mathrm{~cm}$ inner diameter glass tube with a Teflon- sealed cover. The platinum and copper electrodes, sampling point, and gas safety relief valve were inserted into the reactor from the cover, and the two electrodes were placed $5 \mathrm{~cm}$ apart to facilitate operation.

BTEX Characterization. The static head space gas chromatograph method was used to measure organic concentrations. First, the volatile BTEX compounds were extracted with a black fiber using the solid phase microextraction (SPME) technique. ${ }^{3}$ SPME is a small-scale, solventless, and nonexhaustive extraction method developed by Pawliszyn and co-workers. ${ }^{24}$ As our group previously reported, the carboxen/polydimethylsiloxane (CAR/PDMS) fiber showed efficient sample collection. ${ }^{3}$ After SPME preconcentration, the BTEX compounds were desorbed and analyzed by gas chromatography coupled with a flame ionization detector (GC-FID). Potential byproducts formed during the adsorption and electrolysis experiments were investigated by gas chromatography coupled with a mass spectrometry (GC/MS).

A static setup measured the concentration of each individual BTEX compound absorbed by $\mathrm{Fe}_{3} \mathrm{O}_{4} \mathrm{NPs}$. The experiments involved injecting one of the selected BTEX compounds into two flasks to make a 200 ppmv mixing ratio. One flask contained iron oxides NPs (treated flask), and one was without $\mathrm{Fe}_{3} \mathrm{O}_{4} \mathrm{NPs}$ (reference flask). The gas phase mixing ratio of the BTEX compound of the treatment flask was compared to the reference flasks. Reference and treated flasks were systematically evacuated/refilled with dry air at least three times and analyzed by GC-FID before injecting the BTEX compounds. The magnetite and hematite mixtures were added into the flask after vigorous shaking or stirring.

NanoScan Analysis. Hydrodynamic size distribution samples were measured using a Malvern Nanosight NS500 with a laser wavelength of $532 \mathrm{~nm}$ and a EMCCD camera. The capture settings were $29.0099 \mathrm{~ms}$ of shutter length, 309 of camera gain, $17.8 \mathrm{fps}$ of frame rate, and a temperature of $26.6^{\circ} \mathrm{C}$.

\section{Results and discussions}

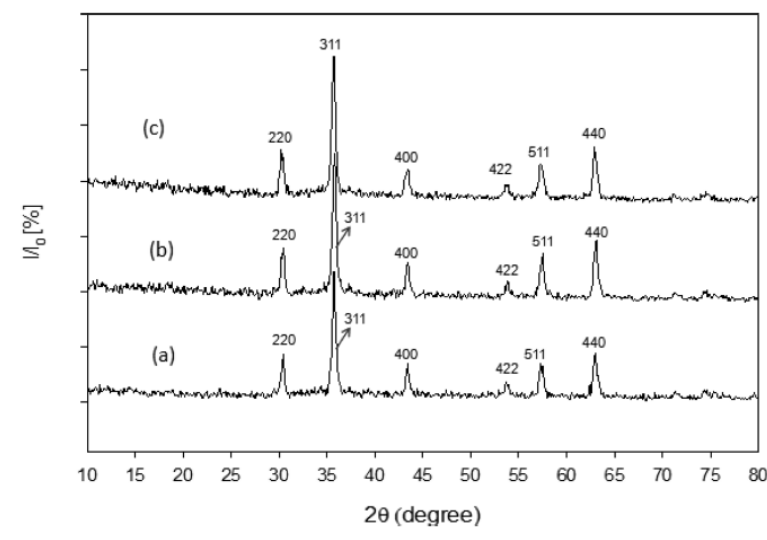

Figure 1. X-ray diffraction patterns of (a) commercial $\mathrm{Fe}_{3} \mathrm{O}_{4} \mathrm{NPs}$ purchased from Sigma-Aldrich, (b) synthesized $\mathrm{Fe}_{3} \mathrm{O}_{4}$ NPs with the Massart ${ }^{23}$ method, and (c) synthesized $\mathrm{Fe}_{3} \mathrm{O}_{4} \mathrm{NPs}$ in this study. 
This document is the unedited Author's version of a Submitted Work that was subsequently accepted for publication in 'The Journal of Physical Chemistry C', copyright @ A American Chemical Society after peer review. To access the final edited and published work: https://pubs.acs.org/doi/abs/10.1021/sc500479b

Characterization of Magnetite NPs. The three types of $\mathrm{Fe}_{3} \mathrm{O}_{4} \mathrm{NPs}$ were characterized together in order to compare their physical and chemical properties. As shown in Figure 1, the XRD relative peak intensities of the commercial $\mathrm{Fe}_{3}$ $\mathrm{O}_{4}$ NPs purchased from Sigma-Aldrich, and synthesized $\mathrm{Fe}_{3} \mathrm{O}_{4}$ NPs matched those of reference $\mathrm{Fe}_{3} \mathrm{O}_{4}$ (JCPDS card no. 19-0629). The average Scherrer sizes of the $\mathrm{Fe}_{3} \mathrm{O}_{4} \mathrm{NPs}$ were 10.2, 7.5, and $6.4 \mathrm{~nm}$. The TEM images (Figure 2) showed that the synthesized $\mathrm{Fe}_{3} \mathrm{O}_{4} \mathrm{NPs}$ had much narrower size distributions than the commercial one. The size of the $\mathrm{Fe}_{3} \mathrm{O}_{4}$ NPs purchased from Sigma-Aldrich, synthesized with the Massart23 method, and synthesized with the modified method in this study ranged from 8 to $50,7-20$, and 8-14 nm, respectively. Because under environmental conditions the nanoaerosol are expected to uptake water, we used a NanoScan instrument, which requires total emersion of particles in ultrapure water to obtain particle distribution. Hence, we obtained an insight on the maximum increase in size of particles once emerged in aqueous media, a situation common in various industrial and environmental applications. NanoScan data showed the same trend as the HR-TEM results; therefore, the improved synthesis method yielded smaller particles. The observed sizes of nanoparticles are expected to be distinct in HR-TEM and $\mathrm{NanoScan}$ analysis due to water emersion in the latter case. Yet, the synthesized $\mathrm{Fe}_{3} \mathrm{O}_{4} \mathrm{NPs}$ systematically exhibit the smallest size distribution (Figure S2, Supporting Information). It should be mentioned that the sizes of the $\mathrm{Fe}_{3} \mathrm{O}_{4} \mathrm{NPs}$ in a water suspension were much bigger than alone due to water uptake by $\mathrm{Fe}_{3} \mathrm{O}_{4} \mathrm{NPs}$. The BET surface areas (SSA) of the commercial $\mathrm{Fe}_{3} \mathrm{O}_{4} \mathrm{NPs}$, synthesized $\mathrm{Fe}_{3} \mathrm{O}_{4}$ NPs with the old method, and synthesized $\mathrm{Fe}_{3} \mathrm{O}_{4}$ NPs with the modified method were 36,57 , and $83 \mathrm{~m}^{2} / \mathrm{g}$, respectively. All of these presented characterization data proved that the modified synthesis method decreased the size, narrowed the size distribution, and increased the BET surface area.

Removal Efficiencies of BTEX by Iron Oxides. One main goal of this study is to investigate the adsorption capacity of iron oxides nanoparticles by measuring removal efficiencies of BTEX compounds. Herein, we used toluene as the model compound. The removal efficiency can be defined as

$$
\text { Removal efficiency }(\%)=\left(\frac{\text { Initial amount of toluene }- \text { Final amount of toluene }}{\text { Initial amount of toluene }}\right) \times 100
$$

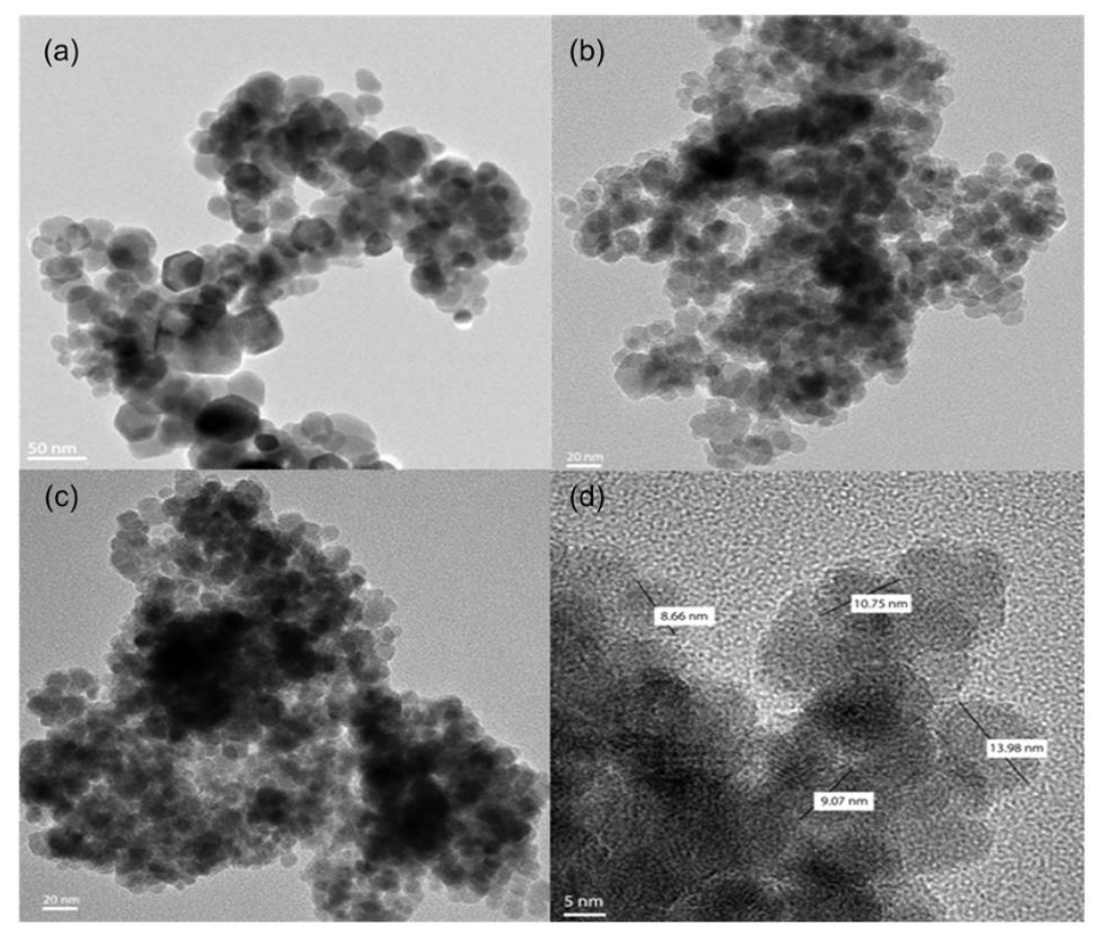

Figure 2. TEM images of (a) commercial $\mathrm{Fe}_{3} \mathrm{O}_{4} \mathrm{NPs}$ purchased from Sigma-Aldrich, (b) synthesized $\mathrm{Fe}_{3} \mathrm{O}_{4} \mathrm{NPs}$ with the Massart ${ }^{23}$ method, and (c, d) synthesized $\mathrm{Fe}_{3} \mathrm{O}_{4}$ NPs in this study.

The previous study in our laboratory experiments proved that $100 \mathrm{ppm}$ of benzene, toluene, ethylbenzene, and $o$ xylene in $2 \mathrm{~L}$ can be removed up to $98 \%$ at room temperature with $4.0 \mathrm{~g}$ of magnetite nanoparticles. We tested the three types of magnetite NPs for the adsorption experiments. We observed that up to 200 ppmv of toluene was removed with an efficiency of $95 \%$ by $1.0 \mathrm{~g}$ of dispersed $\mathrm{Fe}_{3} \mathrm{O}_{4} \mathrm{NPs}$ in $15 \mathrm{~min}$. In order to better compare the adsorption capacity of 
This document is the unedited Author's version of a Submitted Work that was subsequently accepted for publication in 'The Journal of Physical Chemistry C', copyright @ A American Chemical Society after peer review. To access the final edited and published work: https://pubs.acs.org/doi/abs/10.1021/sc500479b

the various $\mathrm{Fe}_{3} \mathrm{O}_{4} \mathrm{NPs}$, the fast adsorption equilibrium should be avoided. Therefore, we decreased the amount of $\mathrm{Fe}_{3} \mathrm{O}_{4}$ NPs and performed the adsorption experiments without stirring the nanoparticles. As presented in Figure 3, the synthesized $\mathrm{Fe}_{3} \mathrm{O}_{4} \mathrm{NPs}$ showed the highest adsorption capacity. The adsorption capacity of the $\mathrm{Fe}_{3} \mathrm{O}_{4} \mathrm{NPs}_{3}$ was dependent on the size and surface area of nanoparticles. The smaller size and larger BET surface area of $\mathrm{Fe}_{3} \mathrm{O}_{4} \mathrm{NPs}$ demonstrated more elevated adsorption capacity. The synthesized $\mathrm{Fe}_{3} \mathrm{O}_{4} \mathrm{NPs}$ also showed the highest adsorption capacities for the other BTEX compounds as well (Table S1, Supporting Information).

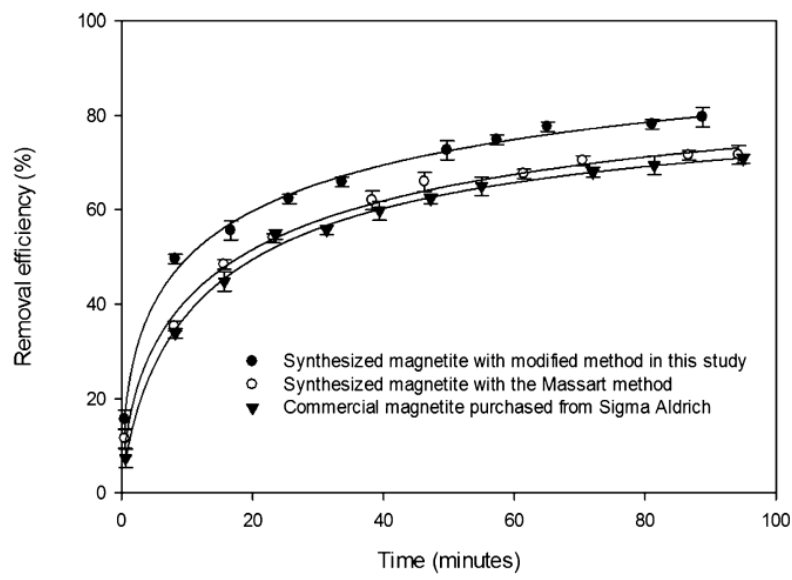

Figure 3. Removal efficiency of toluene adsorption by various $\mathrm{Fe}_{3} \mathrm{O}_{4}$ NPs as a function of time.

Adsorption Capacity Study by $\mathrm{Fe}_{3} \mathrm{O}_{4} / \alpha-\mathrm{Fe}_{2} \mathrm{O}_{3} \mathrm{NPs}$. It has also been reported that hematite $\left(\alpha-\mathrm{Fe}_{2} \mathrm{O}_{3}\right)$ is efficient for gaseous VOCs adsorption. ${ }^{25}$ After evaluating the adsorption of gaseous BTEX on single minerals, we seek to evaluate whether a mixture of minerals could act as the sum of its parts with respect to gas-phase adsorption. The mixture of iron oxides is one of the largest mineral groups on earth. They are ubiquitous in nature and are present in soils, rocks, lakes, and rivers. Magnetite and hematite NPs alone and three different ratios of the mixtures were chosen to test the adsorption capacity. As depicted in Figure 4, the hematite alone showed that the fastest adsorption rate and removal efficiency reached up to $83 \%$ in $1.5 \mathrm{~h}$ with $0.5 \mathrm{~g}$ of NPs. Higher observed removal efficiency can be attributed to the fact that the molecular size of hematite is smaller than magnetite. For a given mass, hematite has more surface-to-volume ratio and consequently more adsorption sites.

For the nanoparticle mixtures, the ones with more amounts of hematite, exhibited faster adsorption rates and better removal efficiency. The experimental values of the mixtures agreed well with the trend, indicating that the iron oxides

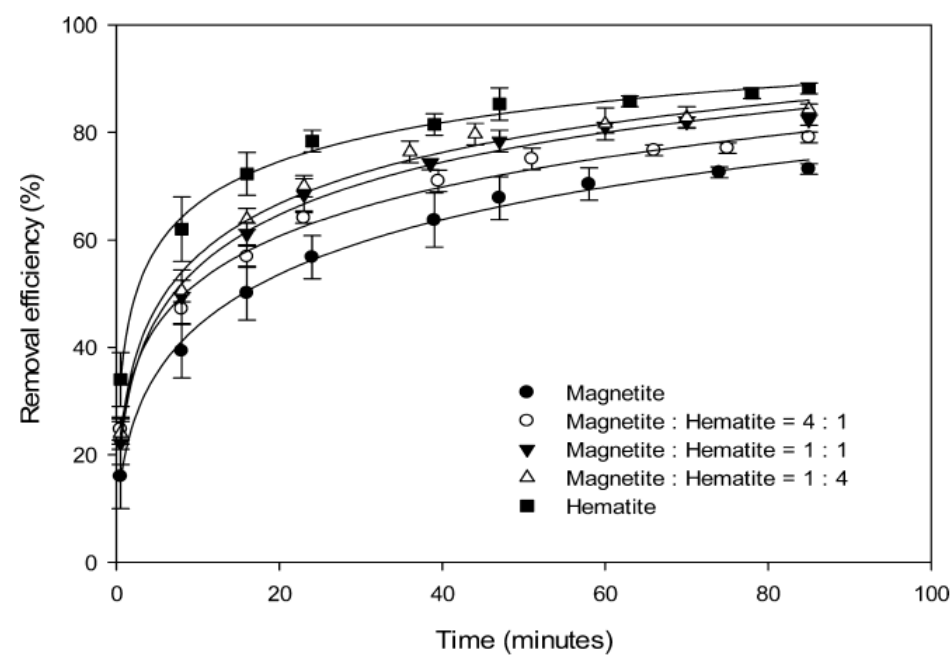

Figure 4. Kinetics of toluene adsorption by magnetite and hematite NPS. 
This document is the unedited Author's version of a Submitted Work that was subsequently accepted for publication in 'The Journal of Physical Chemistry C', copyright @ A American Chemical Society after peer review. To access the final edited and published work: https://pubs.acs.org/doi/abs/10.1021/sc500479b

NPs in the mixture did not interfere with each other as far as adsorption is concerned under experimental conditions. However, we cannot entirely preclude competitive adsorption. To further evaluate the existence of a relationship between the magnetization and adsorption in this study, we measured the magnetization value of the mixture of magnetite and hematite NPs with a 1:1 mixing ratio. The saturation magnetization values of the mixture, before and after toluene adsorption experiments, were 75.8 and $78.0 \mathrm{emu} / \mathrm{g}$, respectively (Figure S3, Supporting Information). The slight observed difference was well within the experimental error (5\%). Nearly identical magnetization values hinted to the fact that the structures of the two NPs did not change with the adsorption process. Further study is encouraged to evaluate whether or not the effect of magnetism is indeed negligible under other environmental conditions. We are indeed optimistic about these results for the application of using naturally abundant iron oxide mixtures as effective adsorbents.

Recovery of BTEX with Different Electrolytes. Monitoring of the stripping efficiency (SE) was used for the assessment of the electrochemical regeneration of nanoparticles. The SE coefficient is defined as follows

$$
S E=\frac{V_{r}}{V_{i}}
$$

where $V_{\mathrm{r}}$ is the amount of toluene desorbed by the magnetite nanoparticles after the regeneration, and $V_{\mathrm{i}}$ is the amount of toluene adsorbed by the magnetite nanoparticles before the regeneration treatment.

Three kinds of simple electrolytes were investigated: (a) sodium chloride, (b) sodium carbonate, and (c) sodium hydroxide. Acidic electrolytes were excluded because of their active reactions with the magnetite. The electrolytes were kept at the same concentration of $0.5 \mathrm{M}$. The electrolysis ran for $2.0 \mathrm{~h}$ with a current of $1.0 \mathrm{~A}$. Although sodiumchloride is relatively inexpensive and more naturally abundant, using neutral $\mathrm{NaCl}$ as the electrolyte had drawbacks because at some point the formation of corrosive chlorine gas would generate toxic chlorinated organics, which damaged the electrodes and SPME fiber. As shown in Figure 5, the $\mathrm{pH}$ value slightly affected the stripping efficiency of toluene. By substituting the neutral electrolyte in lieu of the alkaline electrolyte, the stripping efficiency increased from $80 \%$ to $94 \%$. Results showed that the sodium hydroxide solution exhibited the highest stripping efficiency. Furthermore, we tested the effects of sodium hydroxide without the applied current. The stripping efficiency increased with the increase in the $\mathrm{pH}$ value in the electrolyte solution. The stripping efficiency in the absence of current, however, was no higher than $60 \%$ even when we prolonged the regeneration time up to $8 \mathrm{~h}$.

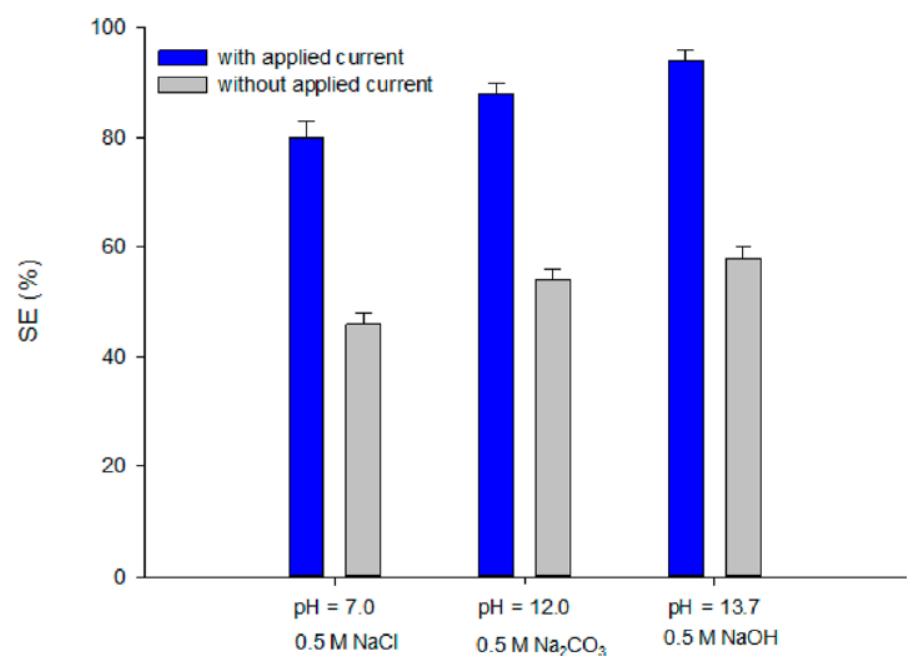

Figure 5. Effect of the electrolyte $\mathrm{pH}$ on the stripping efficiencies (SE).

Recovery of BTEX with Various Current Intensities. To investigate the effect of the current on the electrochemical BTEX regeneration, we used the regeneration of toluene as a model reaction. The electrochemical regeneration experiments were carried out under constant current conditions of $0.25,0.5,0.75,1.0$, and $1.5 \mathrm{~A}$ for $2.0 \mathrm{~h}$ in $0.5 \mathrm{M}$ 
This document is the unedited Author's version of a Submitted Work that was subsequently accepted for publication in 'The Journal of Physical Chemistry C', copyright @ A American Chemical Society after peer review. To access the final edited and published work: https://pubs.acs.org/doi/abs/10.1021/sc500479b

$\mathrm{NaOH}$ (Figure 6). The SE significantly increased from $36 \%$ to $79 \%$ when the current increased from 0.25 to $0.5 \mathrm{~A}$. The SE slightly increased with the increasing current until it reached the highest value, about $94 \%$ at $1.0 \mathrm{~A}$. It has been reported that high electric current and long regeneration time may contribute to the oxidation of the desorbed contaminant to less harmful byproducts. ${ }^{20,22}$ We indeed observed a very small amount of benzaldehyde in our system. As we increased the current up to $2.0 \mathrm{~A}$, there were no significant increases in oxidation products of toluene detected, outside experimental uncertainty limits, nor did significant changes of the stripping efficiencies occur at very high current, yet the stripping efficiency decreased slightly at very high current. As shown in Figure 6, the stripping efficiency slightly decreased from $94 \%$ to $91 \%$ when the current was increased from 1.0 to $1.25 \mathrm{~A}$. These results of iron oxides nanoparticles were similar to those reported in the literature for the regeneration of activated carbon. ${ }^{21,26,27}$ The fast generation of gaseous bubbles corresponding to the high current may deteriorate or block the adsorption/desorption sites. From an energy consumption and economic point of view, it is not beneficial to apply very high current.

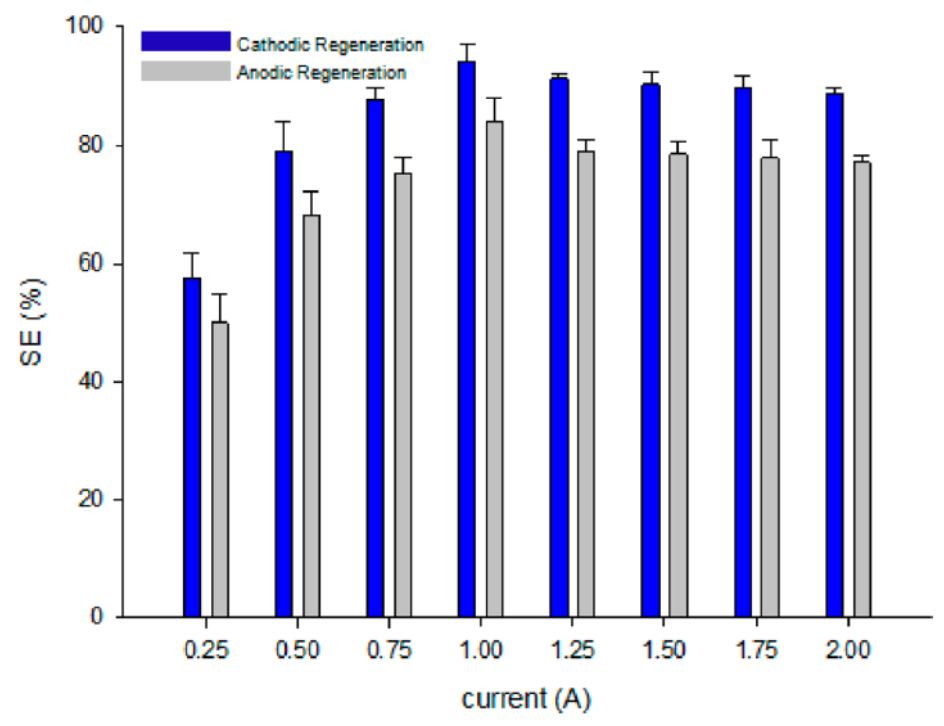

Figure 6. Effect of applied current and electrode polarity on the stripping efficiencies of toluene from $\mathrm{Fe}_{3} \mathrm{O}_{4} \mathrm{NPs}$.

Figure 6 illustrates that for electrochemical regeneration, it is about $10 \%$ more effective to put magnetite nanoparticles in cathodes rather than in anodes. This observation is consistent with previous observations of the cathodic regeneration for exhausted activated carbon saturated with phenol, which has been shown to be more effective than anodic regeneration..$^{21,22,26-28}$ In previous research, the increase in $\mathrm{pH}$ value near the cathode surface destroyed the adsorption equilibrium of phenol in activated carbon. ${ }^{28}$ Meanwhile, the $\mathrm{Na}^{+}$ions moved to the cathode under the influence of the electric field and formed sodium phenate with phenol, which was very difficult to be adsorbed in activated carbon. ${ }^{19}$ Garcia-Otonetal. reported that cathodic regeneration of activated carbon decreased the AC content of oxygen surface groups, which was favorable for the adsorption of toluene ${ }^{20}$ The interaction of BTEX compounds with magnetite depends on the aromatics' basic character and the iron cations' acidic character. ${ }^{3,29}$ As for the regeneration of $\mathrm{Fe}_{3} \mathrm{O}_{4}$ loaded with toluene, we deduced that an elevated ions/radicals (e.g., $\mathrm{OH}$ ) concentration potentially changed the surface charge of the $\mathrm{Fe}_{3} \mathrm{O}_{4} \mathrm{NPs}$, which facilitated desorption of toluene. When the regeneration is performed at the cathode, the $\mathrm{Fe}_{3} \mathrm{O}_{4}$ is subjected to a negative potential, which builds up a negative charge on the nanoparticles, thereby making the toluene more easily desorbed from the $\mathrm{Fe}_{3} \mathrm{O}_{4} \mathrm{NPs}$ and removed by repulsive interactions, thus achieving higher stripping efficiency.

Recovery of BTEX as a Function of Electrolysis Time. The effect of the electrolysis time was also studied. Similar to the effect of the current, the stripping efficiency also increased with the regeneration time. As shown in Figure 7, the stripping efficiency increased dramatically during the first $1.5 \mathrm{~h}$, and after $2.0 \mathrm{~h}$, it slowly reached a constant value. When the electrolysis time was prolonged to $3.0 \mathrm{~h}$, the change in SEcould be ignored. Therefore, all electrolyses ran for $2.0 \mathrm{~h}$ because it was the optimal time to achieve the higher stripping efficiencies. 
This document is the unedited Author's version of a Submitted Work that was subsequently accepted for publication in 'The Journal of Physical Chemistry C', copyright @ A American Chemical Society after peer review. To access the final edited and published work: https://pubs.acs.org/doi/abs/10.1021/sc500479b

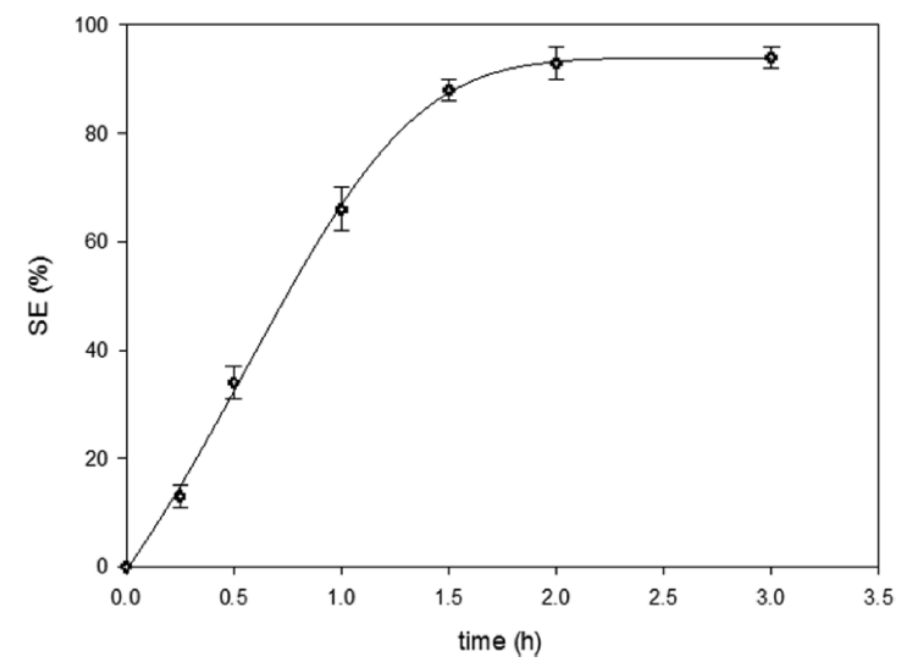

Figure 7. Effect of the regeneration time on $\mathrm{SE}$ of toluene from $\mathrm{Fe}_{3} \mathrm{O}_{4}$ NPs.

Recovery of Various Volatile Organic Compounds. Through the studies described above, the experimental conditions of electrochemical magnetite regeneration were optimized at $1.0 \mathrm{~A}$ for $2.0 \mathrm{~h}$ in a $0.5 \mathrm{M} \mathrm{NaOH}$ medium. On the basis of the optimized reactions, the electrochemical magnetite regenerations loaded with benzene, ethylbenzene, and xylenes were investigated. As shown in Table 1, high stripping efficiencies can also be achieved not only for toluene but also for benzene, ethylbenzene, and xylene. Due to their similar characteristics, toluene and $m$-xylene were expected to exhibit similar electrolysis desorption patterns from the magnetite. The experimental results confirmed our hypothesis (Figure S4, Supporting Information).

Table 1. Effect of Stripping Efficiency vs Adsorbate Type

\begin{tabular}{|ll}
\hline \multicolumn{1}{|c}{ adsorbate } & SE (\%) \\
benzene & $89 \pm 4$ \\
toluene & $92 \pm 2$ \\
ethylbenzene & $93 \pm 1$ \\
$m$-xylene & $95 \pm 2$ \\
$o$-xylene & $94 \pm 1$
\end{tabular}

Regeneration Cycle. It has been reported that activated carbon can be electrochemically regenerated several times without losing its adsorption capacity. ${ }^{20}$ Berenguer et al. ${ }^{21}$ reported that the porosity of granular activated carbon can be well recovered. Our group has recently reported that after the adsorption experiments, nanomagnetite can be thermoregenerated and recycled at least three times after VOC adsorption experiments by collecting them on a magnetic retriever and placing overnight in a $50^{\circ} \mathrm{C}$ air ventilated oven. 3 To assess how the electrochemical treatment protects the magnetite nanoparticles and preserves their adsorption properties, we used regeneration efficiency to compare the adsorption efficiencies of toluene of fresh and regenerated nanomagnetite. As shown in eq 3, the regeneration efficiency can be defined as the ratio of adsorption capacity of regenerated nanomagnetite divided by the adsorption capacity of fresh nanomagnetite.

$$
R E=\frac{\text { Absorption capacity of regenerated nanomagnetite }}{\text { Absorption capacity of fresh nanomagnetite }}
$$

Magnetite loaded with toluene was regenerated up to eight times under optimized conditions of a $0.5 \mathrm{M} \mathrm{NaOH}$ 
This document is the unedited Author's version of a Submitted Work that was subsequently accepted for publication in 'The Journal of Physical Chemistry C', copyright @ A American Chemical Society after peer review. To access the final edited and published work: https://pubs.acs.org/doi/abs/10.1021/sc500479b

electrolyte solution, 1.0 A regeneration current intensity, and $2.0 \mathrm{~h}$ regeneration time. Most experiments were performed under the optimized conditions of a $0.5 \mathrm{MNaOHelectrolyte} \mathrm{solution,} 1.0$ A regeneration current intensity, and $2.0 \mathrm{~h}$ regeneration time. The regeneration efficiency of magnetite nanoparticles was computed. As shown in Figure 8 , the decrease in adsorption capacity of recycled $\mathrm{Fe}_{3} \mathrm{O}_{4} \mathrm{NPs}$ with the adsorption/desorption cycles could almost be neglected under our experimental conditions. It offers potential to upscale the experiments for future industrial use.

We tested the effects of electrochemical regeneration on the physical properties of magnetites. The fresh and regenerated magnetite nanoparticles were characterized by XRD, HR-TEM, and BET analyses. The positions and relative intensities of peaks of regenerated NPs did not change nor did size or shape change based on the TEM images of fresh and recycled $\mathrm{Fe}_{3} \mathrm{O}_{4}$ NPs as depicted in Figure $\mathrm{S} 5$ of the Supporting Information.

The specific surface area of the regenerated NPs was within the allowable experimental error with respect to the fresh ones (Table 2). All results indicated that the electrochemical recycling process did not cause structural damage to the magnetite adsorbent. As summarized in Table 2, the retained physical integrities of the $\mathrm{Fe}_{3} \mathrm{O}_{4} \mathrm{NPs}$ after the recycling procedure suggested that an efficient electrochemical regeneration process of adsorption and desorption for the continuous removal of BTEX is conceivable.

We also investigated the electrochemical regeneration of hematite nanoparticles upon BTEX adsorption. The regeneration efficiency can be up to $90 \%$ under optimized conditions. An example of the results is shown in Figure S6 of the Supporting Information.

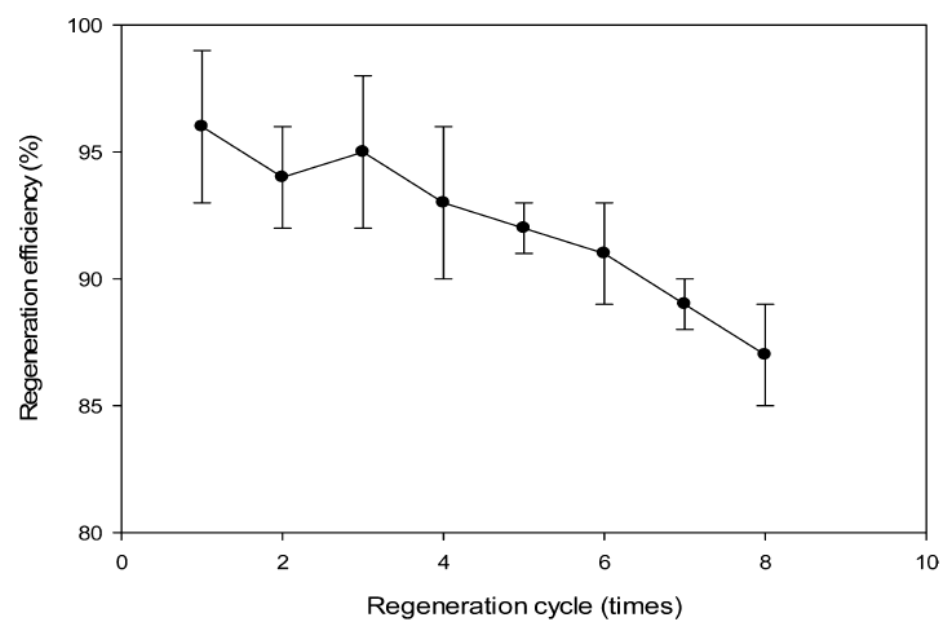

Figure 8. Magnetite regeneration efficiency after different regeneration cycle times.

Table 2. Removal Efficiencies of BTEX with Synthesized and Commercial $\mathrm{Fe}_{3} \mathrm{O}_{4} \mathrm{NPs}$

\begin{tabular}{|c|c|c|c|c|}
\hline adsorbent & $\begin{array}{c}\text { surface area } \\
\left(\mathrm{m}^{2} \mathrm{~g}^{-1}\right)\end{array}$ & size $(\mathrm{nm})$ & $\begin{array}{c}\text { pollutant } \\
(200 \mathrm{ppmv})\end{array}$ & $\mathrm{RE}(\%)$ \\
\hline \multicolumn{5}{|c|}{ synthesized $\mathrm{Fe}_{3} \mathrm{O}_{4} \mathrm{NPs}$ in this study } \\
\hline fresh & $83 \pm 6$ & $8-14$ & $\begin{array}{c}\text { toluene or } \\
o \text {-xylene }\end{array}$ & reference \\
\hline $\begin{array}{l}\text { after three } \\
\text { cycles }\end{array}$ & $76 \pm 6$ & $8-18$ & $\begin{array}{c}\text { toluene or } \\
o \text {-xylene }\end{array}$ & $95 \pm 3$ \\
\hline $\begin{array}{l}\text { after eight } \\
\text { cycles }\end{array}$ & $74 \pm 6$ & $10-20$ & $\begin{array}{c}\text { toluene or } \\
o \text {-xylene }\end{array}$ & $87 \pm 2$ \\
\hline \multicolumn{5}{|c|}{ commercial $\mathrm{Fe}_{3} \mathrm{O}_{4} \mathrm{NPs}$ purchased from Sigma-Aldrich } \\
\hline fresh & $40 \pm 6$ & $18-48$ & toluene & reference \\
\hline $\begin{array}{l}\text { after three } \\
\text { cycles }\end{array}$ & $38 \pm 6$ & $20-46$ & toluene & $94 \pm 2$ \\
\hline $\begin{array}{l}\text { after eight } \\
\text { cycles }\end{array}$ & $33 \pm 6$ & $20-50$ & toluene & $88 \pm 3$ \\
\hline
\end{tabular}


This document is the unedited Author's version of a Submitted Work that was subsequently accepted for publication in 'The Journal of Physical Chemistry C', copyright () American Chemical Society after peer review. To access the final edited and published work: https://pubs.acs.org/doi/abs/10.1021/sc500479b

Potential Reaction Mechanism. Table 1 shows that magnetites can effectively adsorb BTEX. The adsorption process of benzene and monosubstituted benzenes by the magnetite nanoparticles was seemingly rapid due to the external surface adsorption process as well as the microporous adsorption process. ${ }^{30}$ The adsorption mechanism of benzene and monosubstituted benzenes with magnetite nanoparticles has been studied based on acid-base chemistry. ${ }^{29,30}$ The interactions were proposed on cation $-\pi$ interactions between the $\pi$ electrons of aromatic rings and iron cation-terminated activated surfaces. Qualitative calculations suggest a favorable flat configuration of the phenyl group of ethylbenzene onto an $\mathrm{Fe}_{3} \mathrm{O}_{4}$ (111) surface ( $\eta 6$-like ring adsorption geometry). ${ }^{29}$

We herein propose that toluene likely shows similar interactions with magnetite. The calculated heats of toluene adsorption and desorption energy with $\mathrm{Fe}_{3} \mathrm{O}_{4}$ are expected to be similar to the reported data on ethylbenzene (e.g., $E_{\text {des }}\left(\text { ethylbenzene) }=86 \mathrm{~kJ} \mathrm{~mol}^{-1}\right)^{29,31}$ Water molecules can have interactions on the surfaces of $\mathrm{Fe}_{3} \mathrm{O}_{4}$ nanoparticles as well. Extensive studies have been undertaken for water vapor adsorption on magnetite surfaces by exploring an ultra-high vacuum system..$^{32-34}$ The absorption behavior has mainly been reported to occur on two dominant surface sites: $\mathrm{Fe}_{3} \mathrm{O}_{4}(111)$ and $\mathrm{Fe}_{3} \mathrm{O}_{4}(001)$ surfaces. Dissociative, partly dissociative, and coadsorption models are mostly assumed based on the different adsorbed water species with respective saturation cover- age. ${ }^{33,34}$ Laboratory studies have shown that the coexistence of water vapor decreases the $\mathrm{Fe}_{3} \mathrm{O}_{4}$ nanoparticle removal efficiency of toluene. ${ }^{3}$ Kundu et al. have studied the adsorption mechanism of water on magnetite surfaces by atomistic simulation studies. ${ }^{35}$ All of the five different Miller indexed surfaces of $\mathrm{Fe}_{3} \mathrm{O}_{4}(001,011,101,110,111)$, have been used to study the water adsorption behavior in the presence of different numbers of water molecules. Molecular water stabilizes surfaces because surface energy values in the presence of water are lower than pure surfaces.

The adsorption energy in the presence of water molecules on different magnetite surfaces is negative between -60 and $-200 \mathrm{~kJ} \mathrm{~mol}^{-1} .{ }^{35}$ Negative adsorption energy signifies that the thermodynamic stable water interacts with magnetite surfaces. When $\mathrm{Fe}_{3} \mathrm{O}_{4}$ nanoparticles are transferred to an aqueous solution upon toluene, a large population of mobile water molecules surrounds the magnetite nanoparticles. The toluene molecules, which are adsorbed within the magnetite surface, will have to compete with water molecules for the cationic sorption sites of magnetite. Water molecules are expected to bind more strongly to the magnetite active surface sites ${ }^{36}$ It has been reported that 5 or even up to 10 layers of water can be adsorbed on a mineral surface at $90 \%$ relative humidity. ${ }^{25}$ Eventually, the water molecules may thus replace toluene molecules. When magnetite is transferred into the alkaline solution, the magnetite surface will be hydroxylated in nature because hydroxylation shows a very high energy negative reaction. ${ }^{35}$ The probable cause of the $\mathrm{OH}$ adsorption on the magnetite surface is the presence of cations such as $\mathrm{Fe}^{3+}$ or $^{2++}$, which attract negative ions from the solution. Cornell and Schwertmann ${ }^{37}$ have reported that charges on iron oxides arise from the preferential adsorption of hydroxyl ions. The high concentrations of hydroxide in alkaline solution will increase the negative charge density of magnetite particles.

The aromatic phenyl ring can likely produce a region of negative electrostatic potential on the face of the $\pi$ system, which facilitates a natural attraction of $\mathrm{Fe}^{3+}$ cations to the surface. The increasing negative charge density of magnetite particles produces electrostatic repulsion to the toluene molecules and weakens the cation- $\pi$ interactions. Once toluene is desorbed, the hydroxyl ions strongly bind to the cationic sorption sites, preventing the toluene from directly accessing these active sites. On the basis of similar mechanisms, it has been reported that the nanoparticles' dye recovery was conducted by incubating the nanoparticles in acetone. ${ }^{38}$ The dye was released to acetone because of charge competition. The hydrophobic and volatile property of toluene facilitates the desorption equilibrium from the magnet surface in an aqueous solution. More importantly, the electrolysis promotes the electron transfer between $\mathrm{Fe}^{3+}$ and $\mathrm{Fe}^{2+}$ cations and likely stabilizes the active sites. Meanwhile, electrolysis will facilitate the mobility of hydroxide ions and promote the electrostatic attraction with iron cations. At the same time, we cannot exclude the dissolution of the magnetite into the aqueous solution and release of the toluene.

Benzaldehyde, the oxidation product of toluene, has also been found in our electrolysis system using SPME with GC- MS. Although quantification of the oxidation product was not possible, it nevertheless indicates the formation of oxidation compounds, which might be harvested as useful materials in the course of such studies. By optimizing our system, in the future, we may be able to transform the BTEX compounds toless toxic oxidation products.

Chemical reactions of aromatic compounds adsorbed on iron oxides have been reported under harsh reaction conditions, for example, at temperatures greater than $300{ }^{\circ} \mathrm{C} .{ }^{29}$ For example,

the dehydrogenation of ethylbenzene (EB) on $\alpha-\mathrm{Fe}_{2} \mathrm{O}_{3}$ was proposed to occur via $\mathrm{C}-\mathrm{H}$ activation of the ethyl group followed by hydrogen abstraction by basic oxygen in the

oxide..$^{29}$ For our case, it is probably the electronics facilitating the charge arrangement of the cations, which is beneficial for the adsorption of activated oxygen on the surface. The adsorbed activated oxygen on the iron oxides surface will promote oxidation of toluene. Our laboratory has also proven the oxidation of toluene by surface oxygen on $\mathrm{Fe}_{3} \mathrm{O}_{4} \mathrm{NPs}$ when $\mathrm{Fe}_{3} \mathrm{O}_{4} \mathrm{NPs}$ samples were exposed to oxygen upon toluene adsorption. ${ }^{39}$ The electrolysis product 
This document is the unedited Author's version of a Submitted Work that was subsequently accepted for publication in 'The Journal of Physical Chemistry C', copyright () American Chemical Society after peer review. To access the final edited and published work: https://pubs.acs.org/doi/abs/10.1021/sc500479b

of oxygen at the anode can likely promote the formation of benzaldehyde. Meanwhile, the hydroxyl radical formation in the electrolysis, which can initialize the oxidation of toluene, cannot be ruled out.

Cost-Benefit Analysis and Up-Scaling This Novel Technology. To evaluate the potential to up-scale this technique for industrial usage, we considered the costs associated with the treatment of pollutants. Hence, there are key elements that should be considered. For the treatment of gaseous pollutants, the efficient velocity of the operation is essential. According to our experimental results, with removal efficiency up to $95 \%$ in 5 min, the iron oxides adsorption capacity of gaseous BTEX is approximately $1.0 \mathrm{~g} /\left(2.0 \times 10^{-5} \mathrm{~mol}\right)$. Hence, to evaluate the cost, we base our estimation on the treatment of $1.0 \mathrm{~mol} \mathrm{BTEX}$. The mass of iron oxides we need for up-scaling is around $50.0 \mathrm{~kg}$. Ideally, this weight can directly be obtained through naturally occurring iron oxides products. According to sources from the United States Geological Survey Mineral Resources Program, ${ }^{41}$ the average price for the black and red iron oxides in the market is around $1.00 \$ / \mathrm{kg}$. So the cost to treat BTEX is $50.00 \$ / \mathrm{mol}$. It is quite conservative to assume that iron oxides can be recycled up to 10 times using electrochemical procedures as demonstrated. Therefore, the actual cost for the treatment of gaseous BTEX is approximately $5.00 \$ / \mathrm{mol}$. We should mention here that we are only accounting for the cost of the iron oxides samples. The labor costs and other materials such as electrolytes and energy should also be included. We can use natural sea salt as an electrolyte to decrease the cost. The average price for sea salt can be as low as $0.15 \$ / \mathrm{kg} .{ }^{41}$ Meanwhile, we can also use solar energy to supply the electrochemical regeneration, and our preliminary data, not shown in the manuscript, shows that it works well. The cost of the solar-operated equipment is between $\$ 200$ and $\$ 10,000$, and it is a one-time purchase. Due to the magnetic properties of metal-core nanoparticles used in this study, the recovery and transport using magnetic interfaces can significantly decrease the costs in industrial usage. Hence, this sustainable green technology can be operated in an economically modest manner.

\section{Conclusions}

In this study, we developed an efficient green system for the electrochemical regeneration of different iron oxide $\mathrm{Fe}_{3} \mathrm{O}_{4}$ nanoparticles capable of removing BTEX pollutants within a time scale of minutes. These metal core nanoparticles and macroparticles are naturally abundant and inexpensive materials at ambient conditions. Under optimized conditions, the stripping efficiencies obtained for all BTEX compounds were higher than $90 \%$, and the adsorption capacity of magnetite NPs remained constant after several adsorption and electrochemical desorption cycles. We have shown that the efficient electro- chemical regeneration of nanoparticles is feasible. The electrochemical recycling of magnetite intricately depends on the type of the electrolyte, its polarity, electrolysis current, and time. The alkaline electrolyte proved to be the most effective method for the regeneration. Our study indicated higher regeneration efficiency when $\mathrm{Fe}_{3} \mathrm{O}_{4} \mathrm{NPs}$ were placed in cathodes rather than in anodes. At the same time, the stripping efficiency initially increases along with regeneration current intensity and regeneration time until it reached a stable value. Results from our study and others demonstrated that a combination of carboxymethylcellulose-modified magnetite nanoparticles and magnetite nanoparticles are efficient methods for the removal of pollutants, ${ }^{3,40}$ with the advantage that confined interfaces as herein proposed preclude the dispersion of particles in the air stream while avoiding undesirable side products or aerosols, which are known to have adverse effects to climate and air pollution. Further up-scaling studies are encouraged to evaluate the potential of this method for various industrial and environmental endeavors

\section{Associated content}

Supporting Information

Further details on the experimental device and more characterization and experimental results. This material is available free of charge via the Internet at http://pubs.acs.org.

\section{Author information}

Corresponding Author

*E-mail: parisa.ariya@mcgill.ca.

Notes

The authors declare no competing financial interest.

\section{Acknowledgments}

We gratefully acknowledge support of Canadian funding agencies: NSERC, FRQNT (project and PDF fellowship 
This document is the unedited Author's version of a Submitted Work that was subsequently accepted for publication in 'The Journal of Physical Chemistry C', copyright () American Chemical Society after peer review. To access the final edited and published work: https://pubs.acs.org/doi/abs/10.1021/sc500479b

to Z.H.), and Environment Canada. We sincerely thank N.A. Eltouny and U. Shahed for making valuable comments to improve this manuscript.

\section{References}

(1) Hochella, M.F.; Lower, S. K.; Maurice,P.A.;Penn, R. L.; Sahai, N.; Sparks, D. L.; Twining, B. S. Nanominerals, mineral nanoparticles, and earth systems. Science 2008, 319 (5870), 1631-1635.

(2) Snider, G.; Ariya, P. Photo-catalytic oxidation reaction of gaseous mercury over titanium dioxide nanoparticle surfaces. Chem. Phys. Lett.2010, 491 (1-3), 23-28. 
This document is the unedited Author's version of a Submitted Work that was subsequently accepted for publication in 'The Journal of Physical Chemistry C', copyright () American Chemical Society after peer review. To access the final edited and published work: https://pubs.acs.org/doi/abs/10.1021/sc500479b

(3) Eltouny, N. A.; Ariya, P. A. Fe3O4 nanoparticles and carboxymethyl cellulose: A green option for the removal of atmospheric benzene, toluene, ethylbenzene, and o-xylene (BTEX).Ind. Eng. Chem. Res. 2012, 51 (39), 12787-12795.

(4) Chowdhury, S. R.; Yanful, E. K. Arsenic removal from aqueous solutions by adsorption on magnetite nanoparticles. Water Environ. J.2011, 25 (3), 429-437.

(5) Wang, Y. H.; Morin, G.; Ona-Nguema, G.; Juillot, F.; Calas, G.;Brown, G. E. Distinctive arsenic(V) trapping modes by magnetite nanoparticles induced by different sorption processes. Environ. Sci. Technol. 2011, 45 (17), 7258-7266.

(6) Rossi, L. M.; Parize, A. L.; Rubim, J. C. Green synthesis and applications of magnetic nanoparticles. In Handbook of Green Chemistry; Wiley-VCH Verlag GmbH \& Co. KGaA: Weinheim, Germany, 2010.

(7) Chin, S. F.; Pang, S. C.; Tan, C. H. Green synthesis of magnetite nanoparticles (via thermal decomposition method) with controllable size and shape. J. Mater. Environ. Sci. 2011, 2 (3), 299-302.

(8) Vidali, M. Bioremediation. An overview. Pure Appl. Chem. 2001, 73 (7), 1163-1172.

(9) Neff, J. M. Bioaccumulation in Marine Organisms: Effect of Contaminants from Oil Well Produced Water; Elsevier: Amsterdam, The Netherlands, 2002.

(10) Kampa, M.; Castanas, E. Human health effects of air pollution. Environ. Pollut. 2008, 151 (2), 362-367.

(11) Odum, J. R.; Jungkamp, T. P. W.; Griffin, R. J.; Forstner, H. J.

L.; Flagan, R. C.; Seinfeld, J. H. Aromatics, reformulated gasoline, and atmospheric organic aerosol formation. Environ. Sci. Technol. 1997,31

(12) Na, K.; Moon, K.-C.; Kim, Y.P. Source contribution to aromatic VOC concentration and ozone formation potential in the atmosphere of Seoul. Atmos. Environ. 2005, 39 (30), 5517-5524.

(13) Seinfeld, J. H.; Pandis, S. N. Atmospheric Chemistry and Physics:from Air Pollution to Climate Change, 2nd ed.; Wiley: Hoboken, NJ, 2006.

(14) Parmar, G. R.; Rao, N. N. Emerging control technologies forvolatile organic compounds. Crit. Rev. Env. Sci. Technol. 2008, 39 (1), 41-78.

(15) Davis, M. E. Ordered porous materials for emerging applications. Nature 2002, 417 (6891), 813-821.

(16) Plata, D. e. L.; Hart, A. J.; Reddy, C. M.; Gschwend, P. M. Early evaluation of potential environmental impacts of carbon nanotube synthesis by chemical vapor deposition. Environ. Sci. Technol. 2009, 43

(17) Nahm, S. W.; Shim, W. G.; Park, Y. K.; Kim, S. C. Thermal and chemical regeneration of spent activated carbon and its adsorption property for toluene. Chem. Eng. J. 2012, 210, 500-509.

(18) Álvarez, P. M.; BeltránF. J.; Góex-Serrano, V.; Jaramillo, J.; Rodríguez, E. M. Comparison between thermal and ozone regenerations of spent activated carbon exhausted with phenol.Water Res. 2004, 38 (8), 2155-2165.

(19) Zhang, H. Regeneration of exhausted activated carbon by electrochemical method. Chem. Eng. J. 2002, 85 (1), 81-85.

(20) Garcia-Oton, M.; Montilla, F.; Lillo-Rodenas, M. A.; Morallon, E.; Vazquez, J. L. Electrochemical regeneration of activated carbon saturated with toluene. J. Appl. Electrochem. 2005, 35 (3), 319-325.

(21) Berenguer, R.; Marco-Lozar, J. P.; Quijada, C.; Cazorla-Amoroś, D.; MorallónE. Electrochemical regeneration and porosity recovery of phenol-saturated granular activated carbon in an alkaline medium.Carbon 2010, 48 (10), 2734-2745.

(22) Berenguer, R.; Marco-Lozar, J. P.; Quijada, C.; Cazorla-Amoroś,D.; Morallón, E. Comparison among chemical, thermal, and electrochemical regeneration of phenol-saturated activated carbon.Energy Fuels 2010, 24 (6), 3366-3372.

(23) Massart, R. Preparation of aqueous magneticliquids in alkaline and acidic media. IEEE Trans. Magn. 1981, 17 (2), 1247-1248.

(24) Lord, H.; Pawliszyn, J. Evolution of solid-phase microextraction technology. J. Chromatogr. A 2000, 885 (1-2), 153-193.

(25) Goss, K.-U.; Eisenreich, S. J. Adsorption of VOCs from the gas phase to different minerals and a mineral mixture. Environ. Sci. Technol. 1996, 30 (7), 2135-2142.

(26) Narbaitz, R. M.; Cen, J. Electrochemical regeneration of granular activated carbon. Water Res. 1994, 28 (8), $1771-1778$.

(27) Narbaitz, R. M.; Karimi-Jashni, A. Electrochemical regeneration of granular activated carbons loaded with phenol and natural organic matter. Environ. Technol. 2008, 30 (1), 27-36.

(28) Karimi-Jashni, A.; Narbaitz, R. M. Electrochemical reactivation of granular activated carbon: pH dependence. J. Environ. Eng. Sci. 2005, 4 (3), 187-194.

(29) Joseph, Y.; Wühn, M.; Niklewski, A.; Ranke, W.; Weiss, W.; Wöll, C.; Schlögl, R. Interaction of ethylbenzene and styrene with iron oxide model catalyst films at low coverages: A NEXAFS study. Phys.Chem. Chem. Phys. 2000, 2 (22), 5314-5319.

(30) Amin, M.; Bina, B.; Majd, A.; Pourzamani, H. Benzene removal by nano magnetic particles under continuous condition from aqueous solutions. Front. Environ. Sci. Eng. 2013, 1-12.

(31) Zscherpel, D.; Ranke, W.; Weiss, W.; Schlögl, R. Energetics and kinetics of ethylbenzene adsorption on epitaxial FeO (111) and Fe3O4 (111) films studied by thermal desorption and photoelectron spectroscopy. J. Chem. Phys. 1998, 108 (22), 9506-9515.

(32) Joseph, Y.; Ranke, W.; Weiss, W. Water on FeO (111) and Fe3O4 (111): Adsorption behavior on different surface terminations. J. Phys. Chem. B 2000, 104 (14), 3224-3236.

(33) Mulakaluri, N.; Pentcheva, R.; Wieland, M.; Moritz, W.;Scheffler, M. Partial dissociation of water on Fe3O4(001): Adsorbate induced charge and orbital order. Phys. Rev. Lett. 2009, 103 (17),176102.

(34) Mulakaluri, N.; Pentcheva, R.; Scheffler, M. Coveragedependent adsorption mode of water on Fe3O4 (001): Insights from first principles calculations. J. Phys. Chem. C 2010, 114 (25), 11148-11156. 
This document is the unedited Author's version of a Submitted Work that was subsequently accepted for publication in 'The Journal of Physical Chemistry C', copyright () American Chemical Society after peer review. To access the final edited and published work: https://pubs.acs.org/doi/abs/10.1021/sc500479b

(35) Kundu, T. K.; Rao, K. H.; Parker, S. C. Atomistic simulationstudies of magnetite surface structures and adsorption behavior in thepresence of molecular and dissociated water and formic acid. J. Colloid Interface Sci. 2006, 295 (2), 364-373.

(36) Dzade, N. Y.; Roldan, A.; de Leeuw, N. H. A density functional theory study of the adsorption of benzene on hematite $(\alpha-$ Fe2O3) surfaces. Minerals 2014, 4 (1), 89-115.

(37) Cornell, R. M.; Schwertmann, U. The Iron Oxides: Structure, Properties, Reactions, Occurrences, And Uses; Wiley-VCH: Weinheim, Germany, 2003.

(38) Qadri, S.; Ganoe, A.; Haik, Y. Removal and recovery of acridine orange from solutions by use of magnetic nanoparticles. J. Hazard. Mater. 2009, $169(1-3), 318-323$.

(39) Eltouny, N. A.; Ariya, P. A. Competing reactions of selected atmospheric gases on $\mathrm{Fe} 3 \mathrm{O} 4$ nanoparticles surfaces. Phys. Chem. Chem. Phys. 2014, 16 (42), 23056-23066.

(40) Yuan, P.; Fan, M.; Yang, D.; He, H.; Liu, D.; Yuan, A.; Zhu, J.;Chen, T. Montmorillonite-supported magnetite nanoparticles for theremoval of hexavalent chromium [Cr(VI)] from aqueous solutions. J.Hazard. Mater. 2009, $166(2-3), 821-829$.

(41) Minerals Yearbook: Volume I $\square$ Metals and Minerals; MineralResources Progam, United States Geological Survey: Reston, VA,2012. 